\title{
Fluorescence Spectroscopy in Camptothecins Studies
}

\author{
S. KRuszewski ${ }^{a *}$ AND D.M. KRUsZEWSKA ${ }^{b}$ \\ ${ }^{a}$ Medical Physics Division, Biophysics Department, Collegium Medicum of Nicolaus Copernicus University \\ Jagiellońska 13-15, 85-067 Bydgoszcz, Poland \\ ${ }^{b}$ Department of Theoretical Foundations of Biomedical Sciences and Medical Informatics \\ Collegium Medicum of Nicolaus Copernicus University, Jagiellońska 13-15, 85-067 Bydgoszcz, Poland \\ The application of fluorescence spectroscopy methods to determining the properties of analogues of campto- \\ thecin, promising anticancer agents, are described in this paper. The fluorescence anisotropy measurements \\ provide useful information about the binding of camptothecin and its analogues to cell membranes and human \\ serum albumin (HSA) that is important for potential clinical applications of these agents, and permit the selection \\ from many camptothecin analogues those ones exhibiting desirable biomedical properties. Fluorescence anisotropy \\ measurements prove that 3 new camptothecin analogues: 7-tert-butyldimethylsilyl-10-hydroxy-campthothecin, \\ 7-trimethyl-silylethyl-10-hydroxy-camptothecin and 7-trimethyl-silyl-ethyl-10-amino-camptothecin exhibit high \\ affinity of their lactone forms to membranes and low affinity of their carboxylate forms to HSA. Such properties \\ should ensure high stability of these agents in physiological fluids, including blood.
}

PACS numbers: 87.64.kv, 87.15.kp

\section{Introduction}

Camptothecin (CPT) - an alkaloid isolated from the Chinese tree Camptotheca acuminata exhibits very high anticancer activity [1]. CPT is a fluorescent compound and this is a very useful property. Using methods of fluorescence spectroscopy, one can determine or predict the biophysical properties of this promising compound. CPT can exist in two forms: lactone (stable at $\mathrm{pH}<5.5$ ) and carboxylate (stable at $\mathrm{pH}>9$ ). Chemical structures of both forms of camptothecins are presented in Table I. Only lactone form is biologically active. The cellular target of the CPT is topoisomerase I, a nuclear enzyme responsible for DNA replication. CPT interacts only with those cells which are in the S-phase. CPT molecules bind to topoisomerase I - DNA complex and prevent the replication process $[2,3]$. This means that CPT is toxic to the cells that are undergoing DNA synthesis. Cancerous cells are rapidly replicating and they spend more time in the S-phase in relation to healthy tissues, and therefore they are killed with much higher efficiency than the healthy host tissues. Such selectivity of cytotoxicity is a promising property of CPT. Due to the S-phase specificity of CPT, a continuous exposure to this drug must be maintained in order to achieve optimum therapeutic efficacy. Unfortunately, there is some difficulty in fulfilling this requirement. Under physiological conditions $(\mathrm{pH}=7.4) \mathrm{CPT}$ hydrolyses and converts to the "ring opened" inactive carboxylate form [3]. After about $2 \mathrm{~h}$ an equilibrium is achieved and both forms coexist. The concentration of the lactone form in the

* corresponding author; e-mail: skrusz@cm.umk.pl equilibrium is much smaller than the carboxylate form and depends on the environment into which the CPT is introduced. It is about $15 \%, 5 \%$ and $0.2 \%$ for PBS (phosphate buffer saline), whole blood and blood plasma, respectively [4]. The low concentration of the lactone form under physiological conditions seriously limits the possibility of CPT application in cancer chemotherapy. The presence of HSA has a critical influence on the activity of camptothecin. 2 hours after introducing camptothecin to HSA solution, it loses totally its anticancer activity. Camptothecin carboxylate molecules bound to HSA do not convert into the lactone form. This leads to rapid decay of the active lactone form in blood [5]. However, a competing effect exists which improves the stability of camptothecin: CPT molecules bound to membranes do not hydrolyse [6]. Much effort has been undertaken in order to discover analogues of camptothecin which exhibit improved blood stability. Such new compounds should retain anticancer properties and exhibit high affinity of the lactone forms to membranes and low affinity of the carboxylate forms to HSA. Obtaining and study of camptothecin analogues became a big challenge for many laboratories. Hundreds of analogues have been synthesized. Some selected camptothecin analogues are collected in Table I. Among them, camptothecin and topotecan are the most explored agents, especially by the methods of time-resolved fluorescence spectroscopy [6, 7].

The principal subject of this paper is application of steady state fluorescence spectroscopy method to determine the properties of camptothecins: CPT, TPT, and other CPT analogues collected in Table I. The steady state fluorescence anisotropy of above compounds diluted either in HSA solution or membranes suspension was measured and next the binding properties of these agents to HSA and membranes determined. 

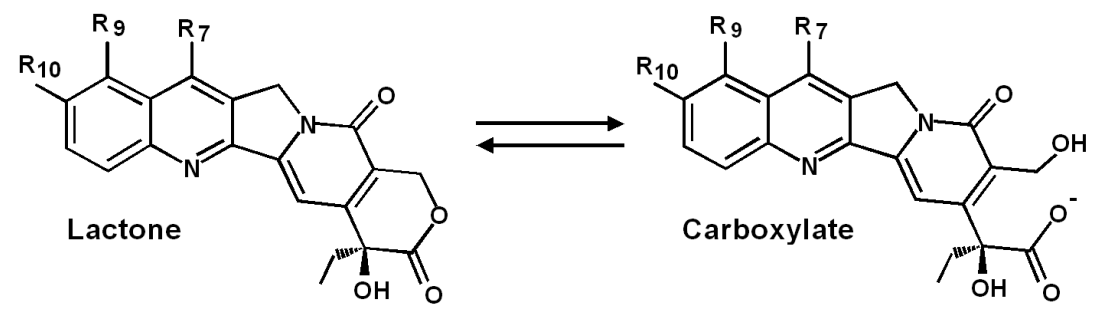

TABLE I

Structure of camptothecin and its analogues.

\begin{tabular}{c|c|c|c}
\hline \hline Compound & $R_{7}$ & $R_{9}$ & $R_{10}$ \\
\hline Camptothecin (CPT) & $\mathrm{H}$ & $\mathrm{H}$ & $\mathrm{H}$ \\
Topotecan (TPT) & $\mathrm{H}$ & $\mathrm{CH}_{2} \mathrm{~N}\left(\mathrm{CH}_{3}\right)_{2}$ & $\mathrm{OH}$ \\
Irinotecan (CPT-11) & $\mathrm{C}_{2} \mathrm{H}_{5}$ & $\mathrm{H}$ & $\mathrm{O}_{2} \mathrm{CNC}_{5} \mathrm{H}_{4} \mathrm{NC}_{5} \mathrm{H}_{5}$ \\
10-OH-CPT & $\mathrm{H}$ & $\mathrm{H}$ & $\mathrm{OH}$ \\
SN-38 & $\mathrm{C}_{2} \mathrm{H}_{5}$ & $\mathrm{H}$ & $\mathrm{OH}$ \\
DB-202 & $\mathrm{Si}\left(\mathrm{CH}_{3}\right)_{2} \mathrm{C}\left(\mathrm{CH}_{3}\right)_{3}$ & $\mathrm{H}$ & $\mathrm{H}$ \\
DB-67 & $\mathrm{Si}\left(\mathrm{CH}_{3}\right)_{2} \mathrm{C}\left(\mathrm{CH}_{3}\right)_{3}$ & $\mathrm{H}$ & $\mathrm{OH}$ \\
DB-174 & $\left(\mathrm{CH}_{2}\right)_{2} \mathrm{Si}\left(\mathrm{CH}_{3}\right)_{3}$ & $\mathrm{H}$ & $\mathrm{OH}$ \\
7-TMSiE-10-A-CPT & $\left(\mathrm{CH}_{2}\right)_{2} \mathrm{Si}\left(\mathrm{CH}_{3}\right)_{3}$ & $\mathrm{H}$ & $\mathrm{NH}_{2}$
\end{tabular}

\section{Materials and methods}

\subsection{Materials}

The samples of camptothecins (camptothecin and its seven analogues) were obtained from the laboratory of biotechnology, College of Pharmacy, University of Kentucky, Lexington, USA. $2 \mathrm{mM}$ stock solutions of camptothecins were prepared in DMSO (dimethylsulfoxide $\mathrm{C}_{2} \mathrm{H}_{6} \mathrm{OS}$ ). Such stock solutions contain only a pure lactone forms. $1 \mathrm{mM}$ stock carboxylate solutions were obtained by dilution of stock lactone solutions in phosphate buffered saline (PBS) at $\mathrm{pH} 12$ in a volume ratio 1:1.

Human serum albumin (95-97\%) was purchased from Sigma-Aldrich (USA - Poland). The solutions of HSA were prepared in PBS. The $\mathrm{pH}$ of these solutions was kept at 7.4 and their temperature at $37^{\circ} \mathrm{C}$.

As model membranes, small unilamelar liposomes formed by DMPC (1,2 dimyristoyl-sn-glycero-3 phosphatidylcholine) lipids were used. DMPC was purchased from Avanti Polar Lipids (Alabaster, AL, USA). Procedure of liposomes preparing was the same as described in previous papers $[8,9]$. For steady state fluorescence anisotropy measurements, the concentration of camptothecins in final samples was equal $2 \mu \mathrm{M}$. The desirable concentration was obtained by adding the stock solutions of camptothecins to HSA solutions at $\mathrm{pH} 7.4$, or to DMPC liposomes suspensions also at $\mathrm{pH} 7.4$.

\subsection{Method and instrumentation}

For characterization of depolarization degree of fluorescence light the fluorescence anisotropy $r$ defined as $r=\frac{I_{\mathrm{V}}-G I_{\mathrm{H}}}{I_{\mathrm{V}}+2 G I_{\mathrm{H}}}[10]$ is most often used. $I_{\mathrm{V}}$ and $I_{\mathrm{H}}$ are the fluorescence intensities of the vertically and horizontally polarized emission, when the sample is excited by vertically polarized light. $G=\frac{S_{\mathrm{V}}}{S_{\mathrm{H}}}$ is the ratio of the sensitivities of the detection system for vertically and horizontally polarized light. On the basis of fluorescence anisotropy measurements in dependence on concentration of lipids forming the model membranes the association (binding) constant of drugs to membranes were determined. The association constant, which is a quantitative measure of affinity of drug to membranes, is determined by formulae [11] $K=\frac{A_{\mathrm{B}}}{A_{\mathrm{F}} L}=\frac{F_{\mathrm{B}}}{F_{\mathrm{F}} L}$, where $A_{\mathrm{F}}$ represents the concentration of free drug, $A_{\mathrm{B}}$ is the concentration of drug bound to membranes and $L$ represents the total concentration of lipids forming membranes. $F_{\mathrm{F}}=A_{\mathrm{F}} / A$ is a fraction of free drug, $F_{\mathrm{B}}=A_{\mathrm{B}} / A$ is the fraction of bound drug and $A=A_{\mathrm{F}}+A_{\mathrm{B}}$ represents the total concentration of drug (the same for all samples).

Procedure of determining the association constant on the basis of fluorescence anisotropy measurements was in details described in previous papers [9, 12].

A PTI (Photon Technology International, Birmingham, NJ, USA) spectrofluorometer in " $L$-format" was used for the measurements of steady-state fluorescence anisotropy. Details of measurements were described previously $[8,9,12]$.

\section{Results and discussion}

CPT do not find clinical application, because of its poor stability in blood caused by high affinity of its carboxylate form to HSA. Topotecan is, beside irinotecan, camptothecin analogue which finds application in therapy of many tumors. In Figs. $1 \mathrm{~A}-\mathrm{C}$ the comparison of properties of CPT and TPT is presented. This comparison was done on the basis of experimental results described in references [6, 7]. Figures $1 \mathrm{~A}$ and $\mathrm{B}$ presents the results of time-resolved fluorescence anisotropy measurements of both forms of CPT and TPT dissolved in HSA solution [6], while Fig. 1C variations in lifetime of fluorescence of both forms of CPT and TPT dissolved also in 
HSA solutions [7]. Figure 1A shows that time-resolved fluorescence anisotropy of CPT lactone decays very fast (rotational correlation time $\Theta_{\mathrm{L}}=90 \mathrm{ps}$ ), while for CPT carboxylate the slow decrease in anisotropy was observed (rotational correlation time $\Theta_{\mathrm{C}}=16 \mathrm{~ns}$ ). It means that the CPT lactone do not bind (or binds poorly) to HSA, while the CPT carboxylate binds strongly to HSA. Figure $1 \mathrm{~B}$ shows the results of similar measurements for TPT. It shows the time-resolved fluorescence anisotropy of both forms of TPT dissolved in HSA solution decreases very fast (rotational correlation time $\Theta_{\mathrm{L}}=\Theta_{\mathrm{C}}=180 \mathrm{ps}$ ), what means that the both forms of TPT do not bind or binds poorly to HSA. Figure $1 \mathrm{C}$ presents how fluorescence lifetime of both forms of CPT and TPT depends on HSA concentration [7]. It shows the CPT lactone and CPT carboxylate behave in different way in HSA solution. Rate of lifetime change of carboxylate CPT is higher than this one of lactone, what proves the CPT carboxylate binds with HSA stronger than CPT lactone. The fluorescence lifetime of TPT for both forms is independent on the HSA concentration, what means that both forms of TPT do not bind with HSA.
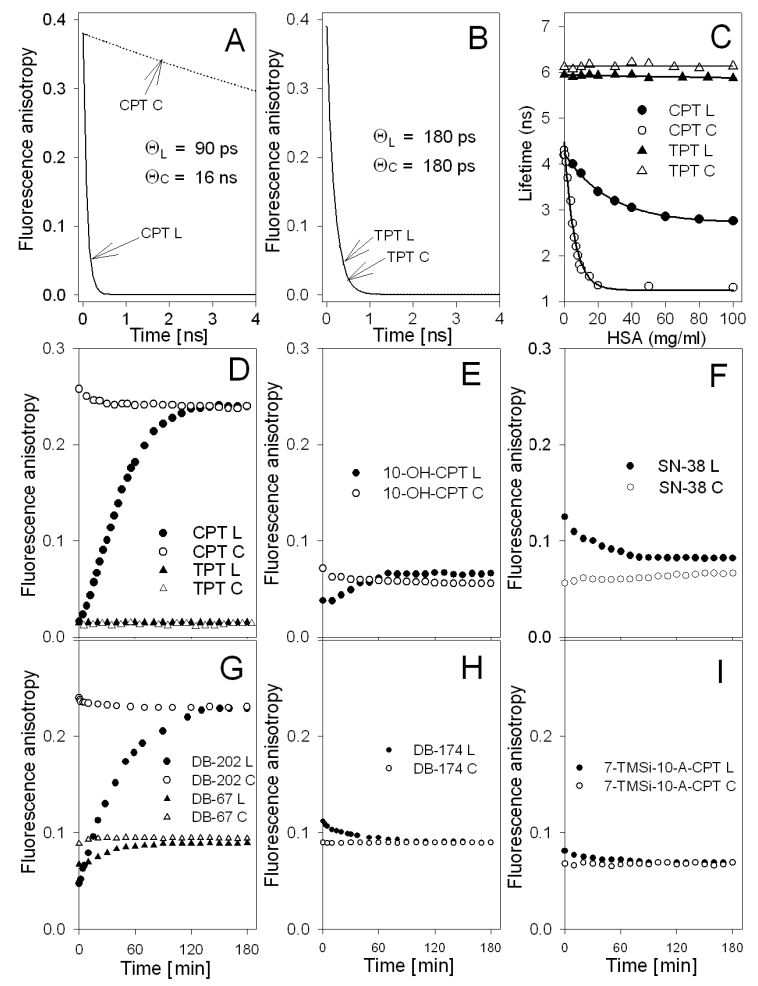

Fig. 1. Comparison of binding properties of camptothecins with HSA. (A) Decays of time-resolved fluorescence anisotropy of both forms of CPT dissolved in HSA solution [6]. (B). Decays of time-resolved fluorescence anisotropy of both forms of TPT dissolved in HSA solution [6]. (C) Fluorescence liftime of both forms of CPT and TPT dissolved in HSA solutions in dependence on HSA concentration [7]. (D-I) Time evolutions of steady-state fluorescence anisotropy of both forms of camptothecins dissolved in HSA solution.
Figure 1D contains the comparison of binding properties of both forms of CPT and TPT to HSA obtained by steady-state fluorescence anisotropy measurements. It shows the steady-state fluorescence anisotropy of the carboxylate form in HSA solution is large and does not change over time, what proves that the molecules of CPT carboxylate are bound to big HSA molecules. CPT lactone just after introduction into HSA solution exhibits low steady-state anisotropy, what means that CPT lactone does not bind or binds poorly to HSA. However, the anisotropy rises over time. This increase is caused by the hydrolysis process. The free CPT lactone molecules convert into carboxylate, which immediately bind to HSA. After about $2 \mathrm{~h}$, as is shown in Fig. 1D, the anisotropy approaches to that obtained for the pure carboxylate form. It means that after such time the lactone form is converted almost totally to inactive carboxylate. In contraire to CPT, TPT behaves totally different in HSA solution. Steady-state fluorescence anisotropy of both forms is small and does not change over time, what confirms earlier conclusions - both TPT forms do not bind with HSA. It is wanted property, because it ensures higher in comparison to CPT stability in blood.
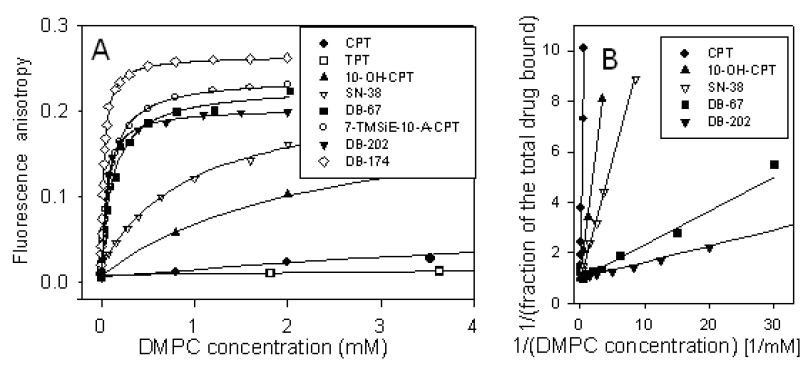

Fig. 2. Steady-state fluorescence anisotropy of lactone forms of CPT, TPT, 10-OH-CPT, SN-38, DB-67, 7-TMSiE-10-A-CPT, DB-202 and DB-174 depending on DMPC liposomes concentration (A) and selected double-reciprocal plots for the binding of the lactone forms of camptothecins to DMPC liposomes (B).

Figures $1 \mathrm{E}$ and $\mathrm{F}$ present the steady-state fluorescence anisotropy of diluted in HSA solution both forms of 10-OH-CPT and SN-38, respectively. These two agents exhibit rather poor affinity of both forms to HSA.

The results of steady-state anisotropy measurements of new camptothecin analogues called silatecans: DB-202, DB-67, DB-174, 7-TMSi-10-A-CPT, are collected in Figs. 1G-I. Figure 1G contains the comparison of binding properties to HSA of DB-202 and DB-67. Molecules, of these compounds, as it is shown in Table I, differs by hydroxyl group at 10 position. This small difference in chemical structure results a big change in binding properties to HSA. Compound without hydroxyl group i.e. DB-202 behaves like CPT in HSA solution. Fluorescence anisotropy of its carboxylate form is high, what means that it exhibits high affinity to HSA. In other hand anisotropy of lactone form is small, but it converts into carboxylate, which immediately binds to 
HSA, what results increase in fluorescence anisotropy. DB-67, which molecule at 7 position contains the same group as DB-202, and additionally at 10 position hydroxyl group, exhibits small and independent on time values of fluorescence anisotropy for both forms. Figure $1 \mathrm{H}$ and I show the fluorescence anisotropy of both forms of DB-174 and both forms of 7TMSi-10-A-CPT (7-trimethyl-silylethyl-10-amino-camptothecin). Molecules of these 2 compounds contain the same group at 7 position, and differ at 10 position having hydroxyl, and amino group, respectively. Results presented in Figs. 1H and I show, that kind of group at position $10\left(\mathrm{OH}\right.$ or $\left.\mathrm{NH}_{2}\right)$ does not influence essentially on binding properties with HSA. Both forms of these 2 agents exhibit poor affinity to HSA.

Overview of binding properties with HSA of CPT analogues permits to conclude that compounds which exhibit poor affinity to HSA (10-OH-CPT, DB-67, DB-174, 7-TMSi-10-A-CPT) may be considered as candidates for further studies including clinical trials, but it will be depend on affinity of their lactone forms to membranes. TPT and practically also SN-38 (it is metabolite of irinotecan) found clinical application.

For quantitative determination of camptothecins binding to membranes the steady-state fluorescence anisotropy over concentration of lipids (which formed liposomes) was measured (Fig. 2A). Rate of increase of fluorescence anisotropy over lipid concentration determines qualitatively value of binding to membranes, then one can conclude that (1) topotecan binds very poorly to membranes, (2) CPT binds also poorly to membranes, (3) 10-OH-CPT and SN-38 bind in medium way to membranes, and (4) silatecans (DB-67, DB-174, 7-TMSi-10-A-CPT, DB-202) exhibit very high binding to membranes.

TABLE II

Summary of the association constants of lactone forms of camptothecins to model membranes (DMPC liposomes) determined using the fluorescence anisotropy method.

\begin{tabular}{c|c}
\hline \hline Compound & $K_{\mathrm{DMPC}}\left[\mathrm{M}^{-1}\right]$ \\
\hline CPT & $70 \pm 15$ \\
TPT & $50 \pm 20$ \\
10-OH-CPT & $560 \pm 80$ \\
SN-38 & $800 \pm 100$ \\
DB-67 & $6600 \pm 1000$ \\
DB-174 & $9000 \pm 1000$ \\
7-TMSiE-10-A-CPT & $5700 \pm 800$ \\
DB-202 & $15500 \pm 1000$
\end{tabular}

On the basis of experimentally determined anisotropies using previously described methods the concentrations of free and bound drugs in liposomes suspension were determined and then the double-reciprocal plots were drawn (Fig. 2B). Slopes of lines fitted to experimental values determine the inverse of association constants $(1 / \mathrm{K})$. Val- ues of associations constants are summarized in Table II. From comparison of binding properties to membranes (Fig. 2 and Table II) with binding properties to HSA (Fig. 1) it follows that 3 silatecans: DB-67, DB-174 and 7-TMSi-10-A-CPT have the desirable properties - their carboxylate forms bind poorly with HSA and lactone forms strongly with membranes.

\section{Conclusion}

Fluorescence anisotropy measurements show that 3 new camptothecin analogues: 7-tert-butyldimethylsilyl-10-hydroxy-campthothecin (DB-67) 7-trimethyl-silylethyl-10-hydroxy-camptothecin (DB-174) and 7-trimethyl-silyl-ethyl-10-amino-camptothecin (7-TMSiE-10-A-CPT) are very promising camptothecin analogues. They exhibit very high affinity of the their lactone forms to membranes and poor affinity of their carboxylate forms to HSA. It means that these agents can become the excellent candidates for further in vivo pharmacological studies, and most probably for clinical trials in cancer chemotherapy.

\section{Acknowledgments}

The authors are very grateful to Lori Latus and Thomas Burke from Tigen Pharmaceuticals Inc. (USA) and the College of Pharmacy, University of Kentucky, Lexington, (USA) for providing the samples of camptothecins.

\section{References}

[1] M.E. Wall, M.C. Wani, C.E. Cook, K.H. Palmer, A.T. McPhail., G.A. Sim, J. Am. Chem. Soc. 88, 3888 (1966).

[2] L.F. Liu, S.D. Desai, T.K. Li, Y. Mao, M. Sun, S.P. Sim, Ann. N.Y. Acad. Sci. 922, . 1 (2000).

[3] K.W. Kohn, Y. Pommier, Ann. N.Y. Acad. Sci. 922, 11 (2000).

[4] Z. Mi, T.G. Burke, Biochem. 33, 10325 (1994).

[5] S. Kruszewski, T.G. Burke, Opt. Appl. 32, 721 (2002).

[6] Z. Mi, H. Malak, T.G. Burke, Biochem. 34, 13772 (1995).

[7] T.G. Burke, Z. Mi, J. Med. Chem. 37, 40 (1994).

[8] S. Kruszewski, D.M. Kruszewska, Opt. Appl. 38, 625 (2008).

[9] B. Ziomkowska, M. Cyrankiewicz, S. Kruszewski, Comb. Chem. High Throughput Screen. 10, 486 (2007).

[10] J.R. Lakowicz, Principles of Fluorescence Spectroscopy. Kluwer Academic/Plenum Publishers, New York 1999.

[11] T.G. Burke, A.K. Mishra, M.C. Wani., M.E. Wall, Biochem. 32, 5352 (1993).

[12] S. Kruszewski, D. Bom, B. Ziomkowska, M. Cyrankiewicz, Opt. Appl. 36, 199 (2006). 